\title{
Comparative Performance Evaluation of Idle Mode Location Management Schemes for IEEE 802.16j Multihop Relay Networks
}

\author{
(Invited Paper) \\ Kuan-Po Lin \\ Institute of Information Science \\ Academia Sinica \\ Taipei, Taiwan \\ gillkuan@gmail.com \\ Hung-Yu Wei \\ Department of Electrical Engineering \\ National Taiwan University \\ Taipei, Taiwan \\ hywei@cc.ee.ntu.edu.tw
}

\begin{abstract}
IEEE 802.16 WiMAX is a promising technology to provide broadband wireless networking access. IEEE 802.16j Mobile Multihop Relay standard introduces multihop relaying to WiMAX system. Relay stations are used to extended network coverage and enhance system throughput. To reduce power consumption, an idle mode MS does not send location update messages while moving across cell borders. Paging mechanism is needed to wake up an idle mode MS. In the $802.16 \mathrm{j}$ multihop relay network, we investigate the performance of several location management schemes. In timer based scheme, an idle mode MS updates when the timer expires. In the paging area based scheme, an idle mode MS updates when moving to a new paging area. In the hybrid scheme, the paging area is recalculated when timer expires. An idle mode updates when it moves across the border of the MS-centric paging area. In this paper, comparative performance evaluation is conducted for these location management schemes with different IEEE 802.16j paging area topologies.
\end{abstract}

\section{Keywords}

IEEE $802.16 \mathrm{j}$, multihop cellular relay network, paging, idle mode, location management

\section{INTRODUCTION}

IEEE 802.16 standard [1] (or WIMAX) is an emerging broadband wireless access system to provide users with high speed

*Corresponding Author. This work was supported by the National Science Council of Taiwan under. Grant NSC-96-2219-E-002-014-

Copyright 2008 ICST 978-963-9799-36-3. multimedia services. The IEEE 802.16e standard [2] provides mobility support for WiMAX system. Power saving for Mobile Stations (MSs) is critical in protocol design, as most an MS is usually powered by battery. Paging mechanism and MS idle mode operation are defined to save power in mobile IEEE 802.16 e system. To reduce power consumption, idle mode MSs do not send location update messages while moving across the cell border.

Recently, IEEE 802.16j Mobile Multihop Relay standards introduce multihop relaying to WiMAX system [3]. Multihop relay communications operates with packet forwarding by Relay station (RS). By applying multihop relay design to centralized WiMAX cellular networks, wireless spectral efficiency could be improved [5]. With relay station deployment, IEEE $802.16 \mathrm{j}$ standard provides better network coverage and enhance system throughput performance. In $802.16 \mathrm{j}$ network, the base station is called Multihop Relay BS (MRBS). Relay Stations forward signaling and data messages between the MR-BS and the MS.

In 802.16 system, an MS enters idle mode to save power when there is no data to be transmitted or to be received. Whenever an incoming data message arrives, the network applies paging mechanism to wake up the dormant MS. During idle mode operation, MS still need to update its location occasionally so that network only needs to perform broadcast paging in selected cells when a data message arrives. Tradeoff between signaling cost and location precision of idle mode MS is the main design issue in idle mode location update mechanism design.

The two basic conventional cellular network paging and location management design are: (1) Location-based paging area schemes[6]: Users update when they move across the border between different paging areas. Paging area might be overlapping or non-overlapping (2)Time-based schemes [4]: Users update periodically when the location update timer expires. The paging and location management in IEEE $802.16 \mathrm{j}$ is not the same as the the traditional cellular systems. There are two types of network nodes, MR-BS and $\mathrm{RS}$, involve in location management. The MS in RS cell transfer data message must pay additional relay cost. Traditional paging scheme whether can have good performance 


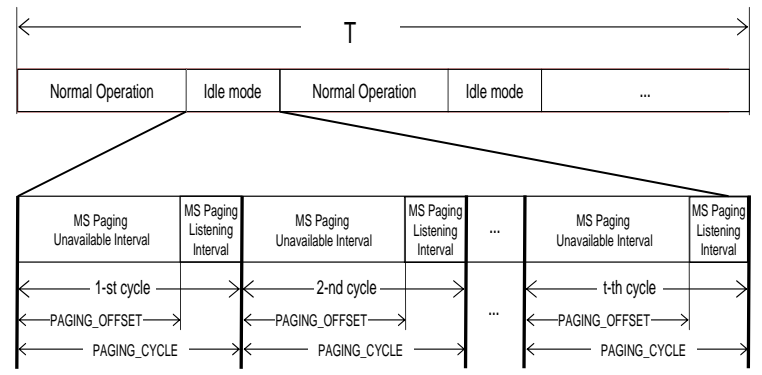

Figure 1: Active mode and idle mode operation

Table 1: Paging and Idle Mode Related Signaling Messages

\begin{tabular}{|c|c|}
\hline Message name & Message description \\
\hline \hline DREG-REQ & SS De-registration message \\
\hline DREG-CMD & De/Re-register Command \\
\hline MOB_PAG-ADV & BS broadcast paging message \\
\hline RNG-REQ & Ranging Request \\
\hline RNG-RSP & Ranging Response \\
\hline
\end{tabular}

in this system is a important issue.

In this paper, we compare the performance in Timer-based and Location-based scheme. In Timer-based scheme, MS update periodically and all MR-BS near MS updated lasted time broadcast messages during listening interval when incoming data messages arrive. In location-based scheme, MS updates when it across the border of the paging area and MR-BS in the same paging area broadcast message when incoming data messages arrive. In addition, we propose a hybrid scheme, the paging area is recalculated when timer expires. An idle mode updates when it moves across the border of the MS-centric paging area. Assume the network is two-hop relay stations in IEEE $802.16 \mathrm{j}$, the evaluation of three schemes are comparative. In the hybrid scheme, the different paging area are selected.

\section{IEEE 802.16J IDLE MODE AND LOCA- TION UPDATE}

Idle mode operation reduces location update signaling. As a result, control signaling cost and MS power consumption are reduced. An MS in idle mode periodically listens to the downlink broadcasting paging messages without registering to a specific BS. RSs relay all paging signaling messages between MS and MR-BS. Idle mode and paging operations are illustrated in Figure 1.

\subsubsection{Entering Idle Mode}

An MS needs to notify the network when it decides to enter idle mode. Before entering idle mode, an MS sends Deregistration message (DREG-REQ) to the MR-BS. Then the MR-BS replies De/Re-register Command message (DREGCMD) to MS. These two signaling messages is used to synchronize the paging listening time. For an MS serving by the relay stations, the access RS will relay all de-registraion messages and paging messages between the MR-BS and the MS. Notice that the control signaling cost is multiplied by the number of relay hops in this scenario.

\subsubsection{Idle Mode Operation}

There are two types of time intervals in idle mode operation: MS Paging Unavailable Interval and MS Paging Listening Interval. During MS Paging Unavailable Interval, an MS turns off radio interface to save power. In MS Paging Listening Interval, an MS listens to the downlink broadcast of paging advertisement messages (MOB_PAG-ADV). An MS is synchronized to the periodic listening intervals based on the paging cycle parameter and paging offset parameter specified by MOB_PAG-ADV messages. The listening interval operates periodically with paging cycles. The paging offset parameter is used to classify MSs into different paging groups. Each paging group will listen to broadcast paging from the beginning of the paging offset duration in each paging cycle.

\subsubsection{Termination of Idle Mode}

At the end of an MS listening interval, the MS must decide whether to leave idle mode or not. If an MS would like to transmit data, it must leave idle mode and enter active mode for normal operation. When an MS decides to enter active mode, it will start the network re-entry process. The network re-entry process is similar to the initial network entry process by first sending Ranging Request (RNG-REQ) message to the serving MR-BS. Then MR-BS will reply with Ranging Response (RNG-RSP) message to the MS. The MS can send the location update message and start the active mode operation. RSs will forward signaling messages, such as RNG-REQ and RNG-RSP, between MS and MR-BS.

The paging operation is initiated when the system want to find an idle mode MS. For example, a new data packet is arrived and is to be delivered to the MS in idle mode. The network will check the paging information database that records the associated paging group of the to-be-paged MS. All MR$\mathrm{BS}$ and access relay stations in the paging group will send broadcast paging message MOB_PAG-ADV with the MS's MAC address. Once the MS receives the broadcast paging message, it will terminate the idle mode and go back to normal mode. The MOB_PAG-ADV broadcasting is initiated from the MR-BS and is forwarded through relay stations.

\section{LOCATION UPDATE MECHANISM FOR IEEE 802.16J IDLE MODE}

IEEE $802.16 \mathrm{j}$ standard defines the protocol signaling flows and the signaling message format for idle mode operations. However, the location update algorithm and paging algorithm are implementation issues. The wireless service providers are free to choose the idle mode location update algorithms, as long as the implemented algorithms use the standardized signaling message format and are compatible with the defined protocol signaling flows. In this paper, we compare the performance of the following three types of location update schemes

- Timer based location update

- Paging area based location update

- Hybrid location update 


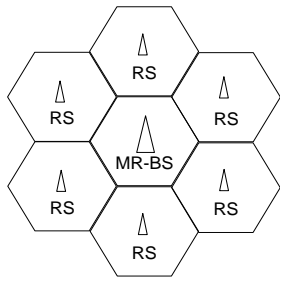

Figure 2: IEEE 802.16j multihop cellular structure: base stations and relay stations

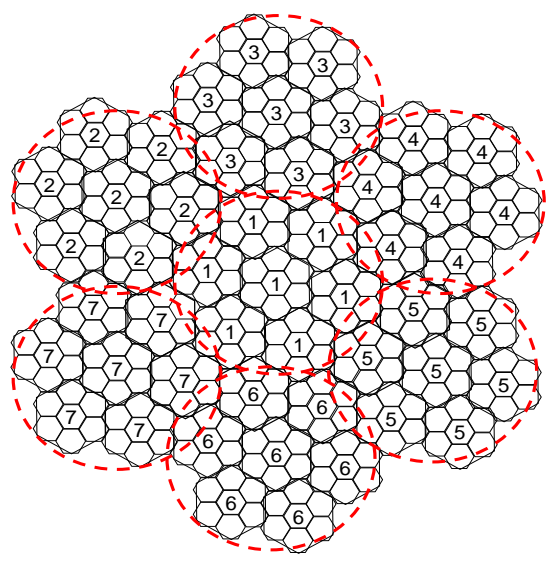

Figure 4: The original paging area scheme

In timer based scheme, an idle mode MS sends location update messages when its location update timer expires. In the paging area based scheme, an idle mode MS sends location update messages when moving to a new paging area. In the hybrid scheme, an MS-centric paging area based update and timer based update are both applied. The MS-centric paging area is reset when the MS sends a location update message. An idle MS sends a location update and then recalculated its paging area if it has not updated for more than a update timer value. An idle mode MS also sends a location update message when it moves across the border of the MS-centric paging area.

\subsection{Hybrid Location Update Scheme}

The hybrid paging scheme integrates timer based mechanism and paging area based mechanism. The paging area recalculate when the time expire. The paging areas can have many choices. The MS roaming in the area during idle mode operation and three types of events might occur: Timer-Based Paging Area Update, Broadcast Paging, and Paging Area Notification (PA Notification). These events occur according to the MS random walk mobility model and the data messages arriving process.

\subsection{MS-Centric Paging Area in Hybrid Scheme}

An IEEE $802.16 \mathrm{j}$ multihop cell might be composed of one MR-BS and multiple relay stations. For example, a twohop multihop 802.16j cell is shown in Fig. 2. There is one MR-BS and 6 relay stations that are connected to MR-BS directly. Each paging area is composed of several multihop cells. Based on this two-hop multihop cellular structure, eight paging area configurations for hybrid location update schemes are illustrated in Fig. 3. The multihop cells in the same paging area are labelled with the same number. The centered hexagonal that labelled with the paging area number is with an MR-BS. Other non-labelled hexagonals are with RSs, which connect to the closest MR-BS.

These paging area configurations could be classified into onetier paging area configuration and two-tier paging area configuration. As the terminology suggested, any paging area in one-tier paging area configuration has one tier of multihop cell. Likewise, any paging area in two-tier paging area configuration has two tiers of multihop cells.

As described previously, when an idle mode MS updates its location, the MS-centric paging area is recomputed. The current cell is relabelled as the central cell in the updated paging area. In each type of paging area configuration, paging areas can be divided into 60 degree, 120 degree, 180 degree, and 360 degree, as shown in Fig. 3. With a large paging area, an idle mode MS updates its location less frequently as it move across the border of a paging area less often. With a small paging area, the broadcast paging cost is low when the network need to broadcast the whole paging area to wake up an idle mode MS.

\subsection{Paging Area Configuration in Paging Area Based Scheme}

Unlike the paging area configuration in hybrid scheme, where paging area configuration is symmetric to the central cell, the paging area configuration in paging area based scheme repeats a regular pattern. The paging area configuration for paging area based location update scheme is shown in Fig. 4. Notice that there is no paging area configuration needed for a timer based location update scheme, in which an idle mode MS only updates its location when its update timer expires.

\section{SIGNALING COST FOR IDLE MODE OP- ERATION}

The main differnce between the IEEE $802.16 \mathrm{j}$ multihop cellular network and the conventional network is that IEEE $802.16 \mathrm{j}$ applies multihop relay. In the paging and location update signaling message analysis, we need to consider the additional signaling cost due to multihop relay. The signaling cost for location update and paging in IEEE $802.16 \mathrm{j}$ idle mode operation could be classified into three types.

- Timer-Based Location Update Signaling

- Broadcast Paging Signaling

- Paging Area Notification Signaling

\subsection{Timer-Based Location Update}

Timer-based location update (Timer-Based PA Update) occurs when the update timer $t$ expires. The system recomputes the MS-centric paging area in the hybrid location update scheme. The MS located cell is the new central cell in the MS-centric paging area configuration. In addition, the same MS-centric paging area re-computation occurs when 

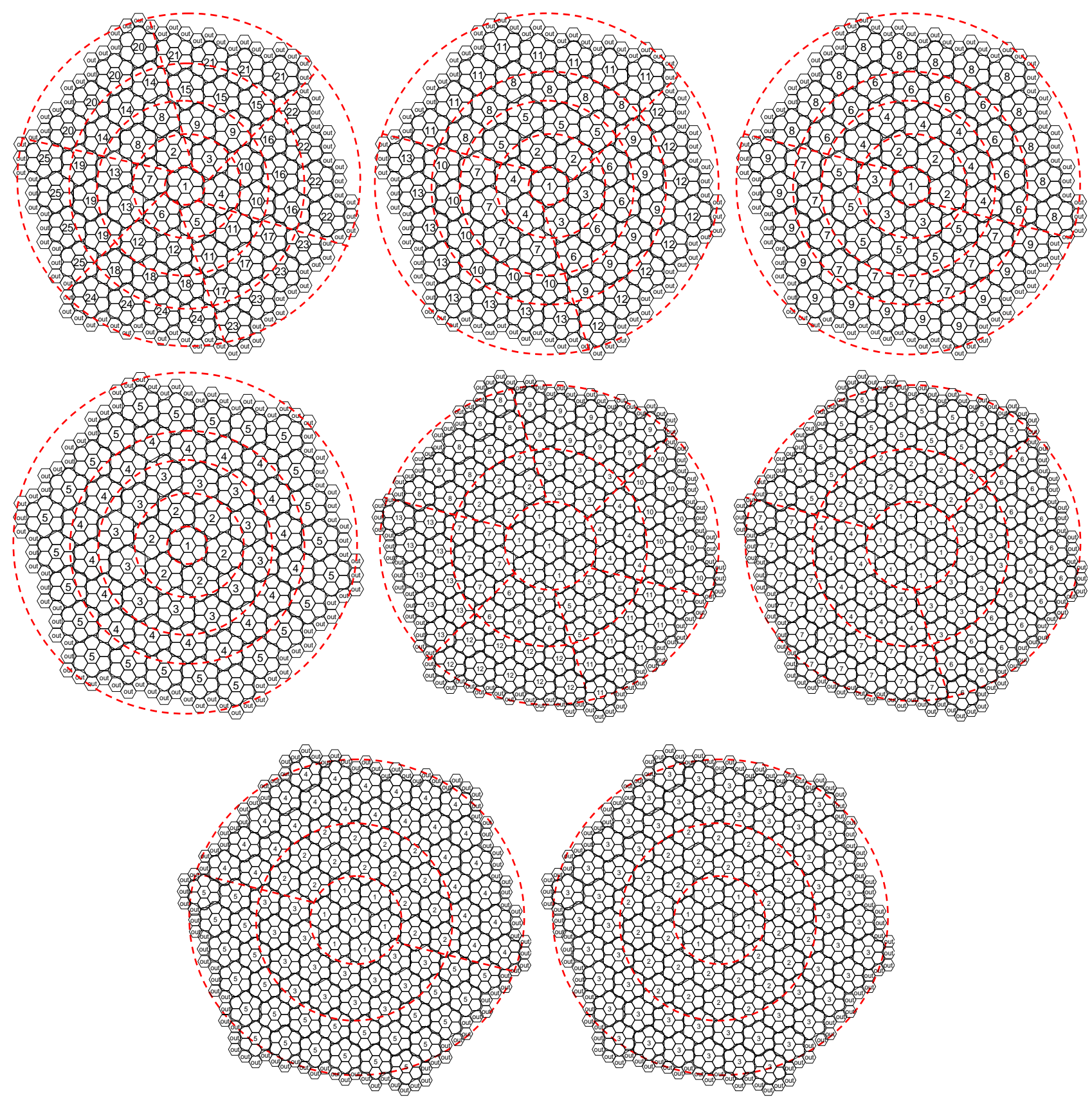

Figure 3: Paging Area Topologies in Hybrid Scheme (a)One-Tier $60^{\circ}$ (b)One-Tier $120^{\circ}$ (c)One-Tier $180^{\circ}(\mathrm{d}) \mathrm{One}-$ Tier $360^{\circ}$ (e)Two-Tier $60^{\circ}$ (f)Two-Tier $120^{\circ}$ (g)Two-Tier $180^{\circ}$ (h)Two-Tier $360^{\circ}$ 
a MS goes into active mode, which happens after a data message arrives. In paging area based scheme, there is no timer-based PA update cost. In timer based scheme, the timer-based location update occurs periodically.

\subsection{Broadcast Paging}

Broadcast paging event only occurs when the data message arrives. If the call arrives, the system broadcasts a paging message to the paging area where the MS locates. In the timer based scheme, the paging broadcast is conducted in all possible cells around the last location update location.

\subsection{Paging Area Notification (PA Notification)}

If the MS moves across the border between two different paging areas, the MS must notify the network about the PA change. In both paging area based location update scheme and the hybrid scheme, the PA Notification signal cost occurs when an idle mode MS moves across the paging area border. However, the PA Notification in paging area based scheme is the update cost of crossing fixed paging area boundaries. On the contrary, the PA Notification in the hybrid scheme is the update cost of crossing MS-centric paging area boundaries. There is no PA Notification cost in timer based scheme.

\subsection{Minimizing Signaling Cost}

In the hybrid location update scheme, both timer based update and paging area update are conducted. The location update timer $t$ could be optimized so that the total signaling cost will be minimal. As the update period increases, the Timer-Based PA Update signaling cost reduces. As the paging area configuration shown in Fig. 3, the paging area is smaller near the center. As the update period decreases, the Broadcast Paging signaling cost reduces. Moreover, the PA Notification signaling cost reduces as the update timer value increases. The optimized update timer $t^{*}$ could be derived after taking these three signaling cost components into consideration.

\section{PERFORMANCE EVALUATION}

In this section, we compare performance of the location update schemes for IEEE 802.16j idle mode operations. Different paging area configurations in the hybrid location update scheme are also evaluated. We also investigate the optimized location update timer to minimize the signaling cost. The discrete time simulator is written by $\mathrm{C}++$. The simulation parameters include the data message arrival rate $\lambda$ that characterizes the probability of an idle mode MS entering active mode. The MS mobility is modelled by the random walk mobility model in the hexagonal topology. The mobility probability is denoted as $p$, which is the probability that an idle mode MS stays in the same cell at the beginning of the next time slot.

\subsection{Optimized Location Update Timer In Dif- ferent Mobility Scenarios}

In Fig. 5 and 6 , we choose the same message arrival rate $\lambda=$ 0.05 to investigate the optimal update timer value $t^{*}$ and total signaling cost in the hybrid location update scheme. The one-tier paging area configuration is used. When the mobility probability $p$ increases, which indicates idle mode

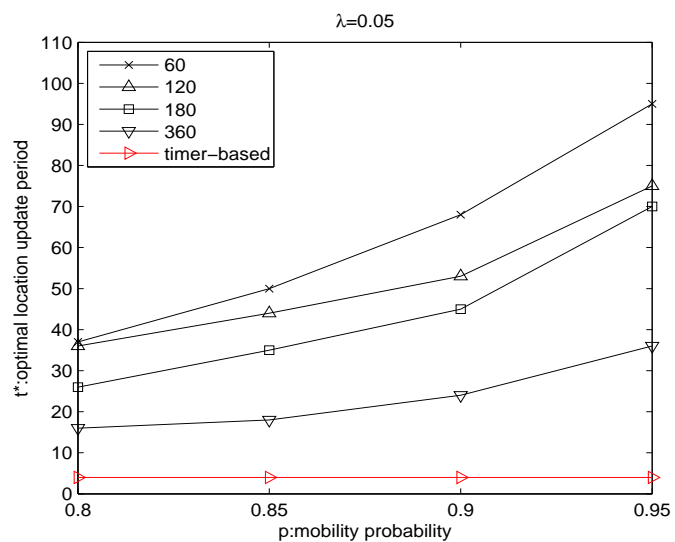

Figure 5: The optimal update period with different mobility probability parameters

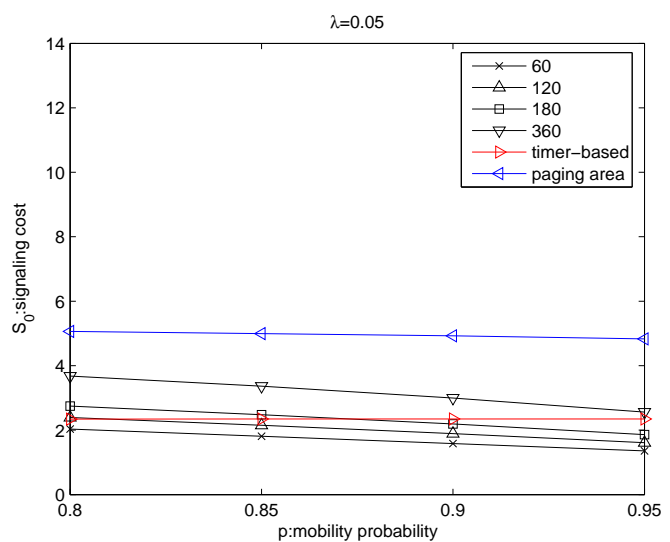

Figure 6: The signaling cost with different mobility probability parameters

MSs moves less frequently, the optimal $t^{*}$ increases. This is intuitive as we do not need to update idle mode MS location frequently in a low mobility scenarios. In addition, we can observe that the total signaling cost is lowers in low mobility scenario.

The $t$ of the timer based location update scheme is small to keep track of the idle mode MSs. The hybrid scheme can achieve lower signaling cost when the mobility probability $p$ become larger. The signaling cost of the paging area based scheme does not change much when MS's mobility parameter changes, as the paging area based scheme does not optimize with the update period. We also compare four different paging area configurations in the hybrid scheme. The 60 degree configuration is the best. The paging area is small in this case. Location update and paging broadcasting signaling cost in the 60 degree configuration is low.

In Fig. 7, 8, we use two-tier paging area configurations during simulation. We also compare the optimal $t^{*}$ and the total signaling cost.The 60 degree paging area configuration 


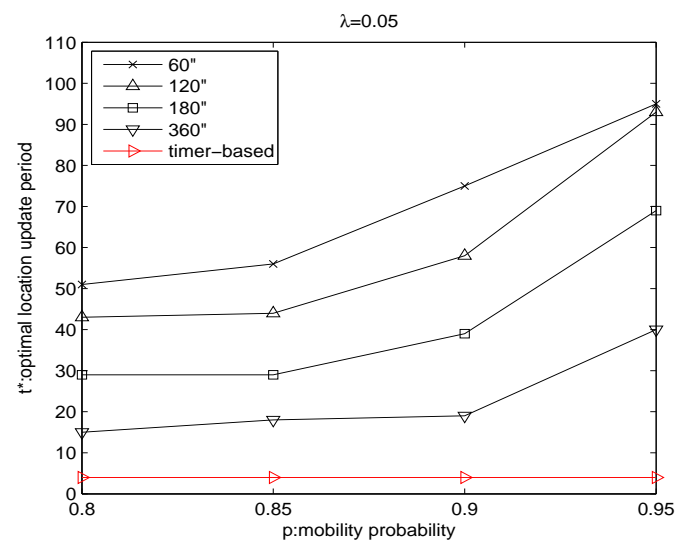

Figure 7: The optimal update period with different mobility probability parameters in two-tier paging area configuration

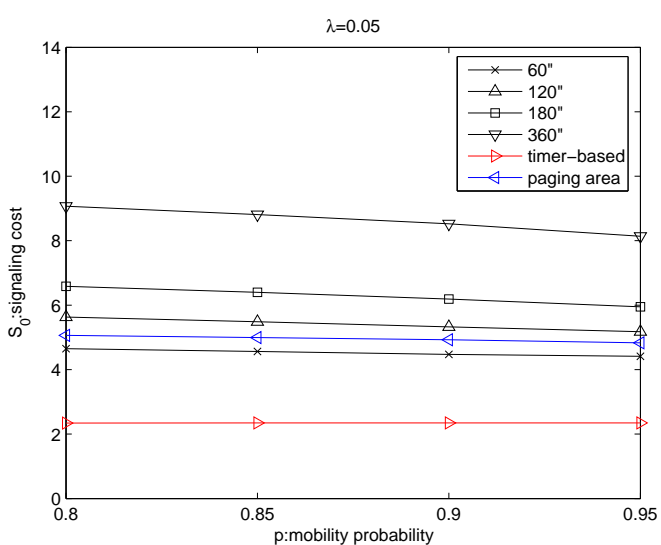

Figure 8: The signaling cost with different mobility probability parameters in two-tier paging area configuration

is the best among hybrid location update schemes. However, the comparative performance to the timer based location update is poor.

\subsection{Effect of Message Arrival Rate}

In Fig.9 and 10, we select the fix mobility probability $p=$ 0.09 and observe the system performance in scenarios with different message arrival rate $\lambda$ parameters. When the message arrival rate increases, the total cost increases. In this case the optimal $t$ decreases because the paging area is recalculated after paging broadcasting that follows the data message arrival.

We can observe that the optimal update timer value decreases as the message arrival rate decreases. As an idle mode MS enters to active mode more frequently, the location is updated more frequently while entering active mode. On the other hand, the overall signaling cost increase with the message arrival rate because of the increasing number of

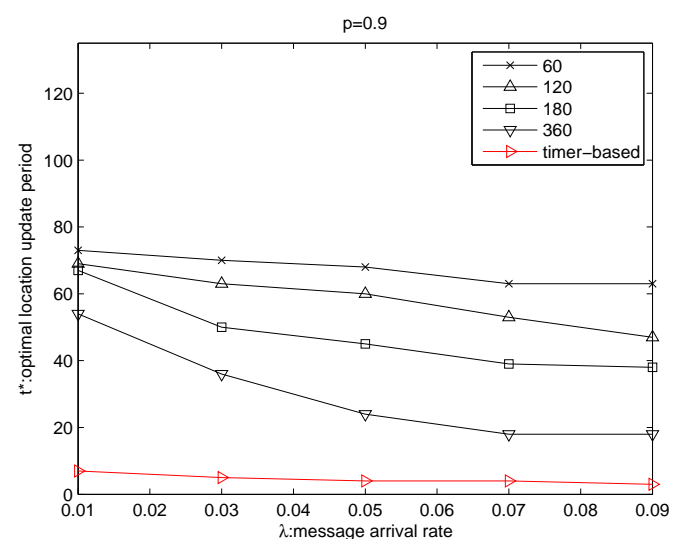

Figure 9: The optimal update period with different message arrival rates

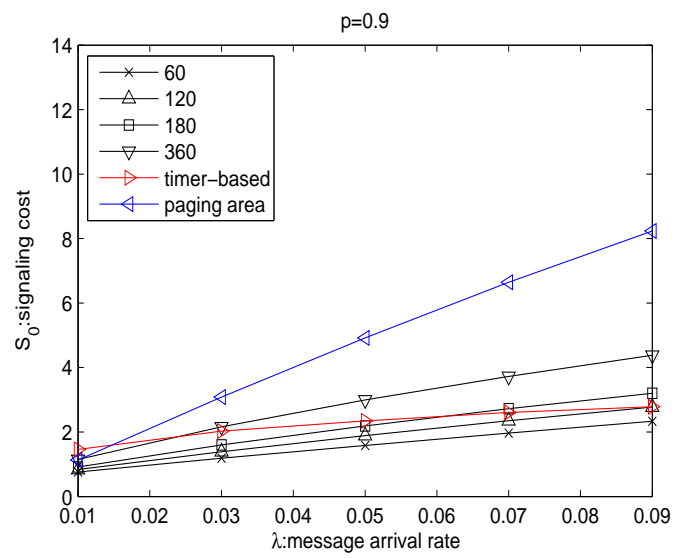

Figure 10: The signaling cost with different message arrival rates

broadcast paging events.

Comparing the optimal update period $t^{*}$ in the hybrid schemes, the 60 degree configuration has the lowest $t^{*}$ value. As a result, the timer-based update signaling cost is also lower because of the low update frequency. In this scenario, even though the $t^{*}$ of the pure timer based scheme is small, the hybrid scheme with the 60 degree configuration is better due to the hybrid paging area based and timer based update. The pure paging scheme can have better performance when the message arrival rate is low. However, in most of the cases, the paging area based update scheme perform worse than all other update schemes. The main reason of such high signaling cost in the paging area based update scheme is that the broadcast cast paging cost is high, compared with the location update cost. Hence, when message arrival rate is low, the total signaling cost is significantly lower.

As shown in Fig.11 and 12, we also compare the performance with the two-tier paging area configuration. The optimal t in 60 degree is larger than other paging area configurations. The total cost is also the lowest among the hybrid schemes. 


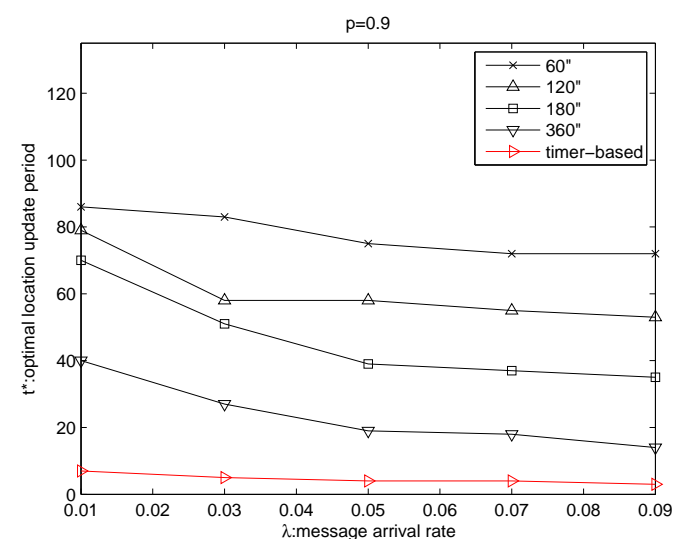

Figure 11: The optimal update period with different message arrival rates in two-tier paging area configuration

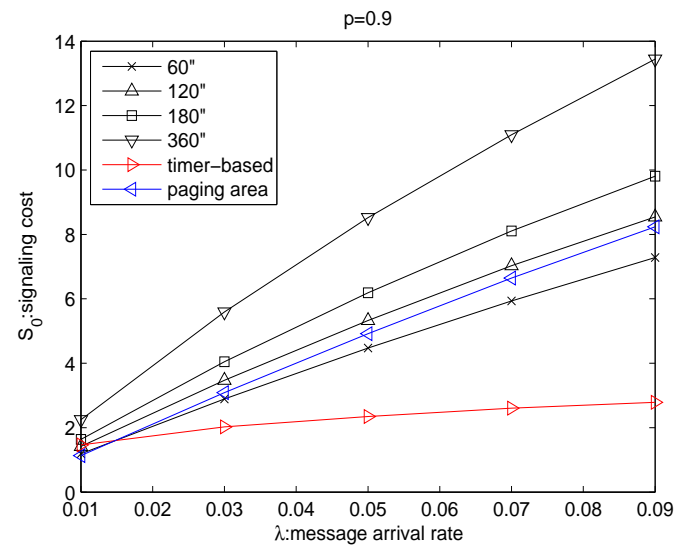

Figure 12: The signaling cost with different message arrival rates in two-tier paging area configuration

The timer based scheme is better than all the hybrid location update schemes with two-tier paging area configurations. The hybrid location update scheme with one-tier paging area configurations have shown better performance over the two-tier configurations.

\subsection{Composition of Signaling Cost}

The overall signaling cost is composed timer-based location update cost, broadcast paging signaling cost, and paging area notification signaling cost. The three types of signaling cost for one-tier and two-tier 60 degree configurations are shown in Fig. 13 and 14 respectively. The $\lambda$ of these two cases are the same, so the frequency of broadcast paging are the same. Since the size of paging areas are different, the broadcast paging cost are different. Since the size of the paging area in the one-tier 60-degree configuration is quite small, the frequency of an MS moving across the PA boundary is high. In the one-tier scenario, the PA notification cost is higher, compared with the case in two-tier scenario. The total signaling cost in the two-tier case is lower than the cost

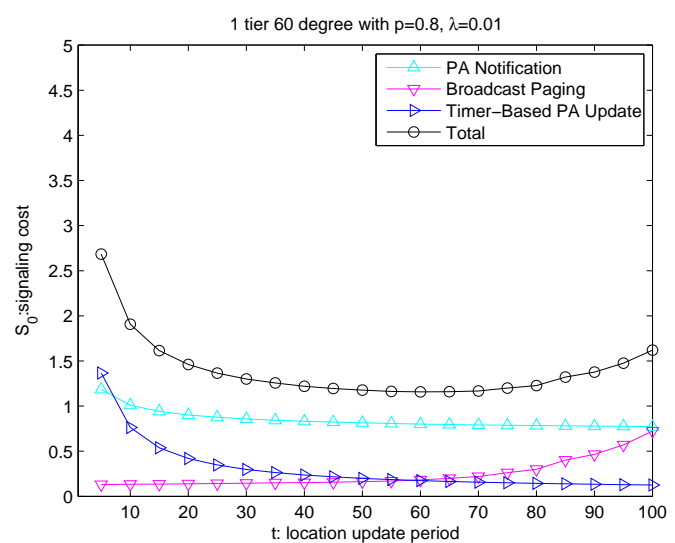

Figure 13: The three types of signlaing cost of onetier 60 degree paging area configuration

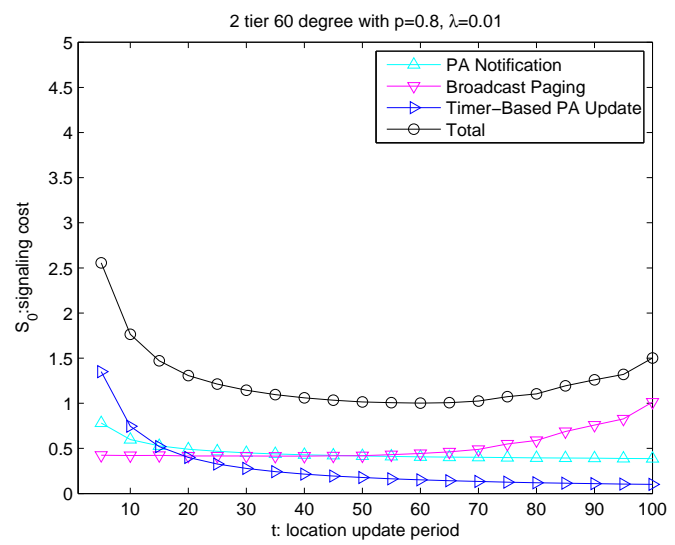

Figure 14: The three types of signlaing cost of twotier 60 degree paging area configuration

in the one-tier case.

\subsection{Operation in Very Low Arrival Rate Sce- nario}

As shown in Fig. 14, the performance results of the evaluated location update schemes are around the same level when the message arrival rate $\lambda=0.01$. We are interested in finding the performance results when $\lambda$ is less than 0.01 . In Fig. 15 and 16, we compare the performance results with the very low message arrival rate. The paging area based scheme performs well in low $\lambda$ scenarios. On the contrary, the timer based scheme does not perform well. When $\lambda$ is small, the broadcast paging cost is low. With small $\lambda$, the main factor of the signaling cost is due to paging area notification cost in the paging area based scheme and the hybrid update schemes. Comparatively, the paging area in the hybrid scheme is larger than the paging area in the paging area based update scheme; hence, the paging area based update scheme performs better. On the other hand, even though data message arrives in frequently, the timer based update scheme needs to transmit update signaling messages period- 


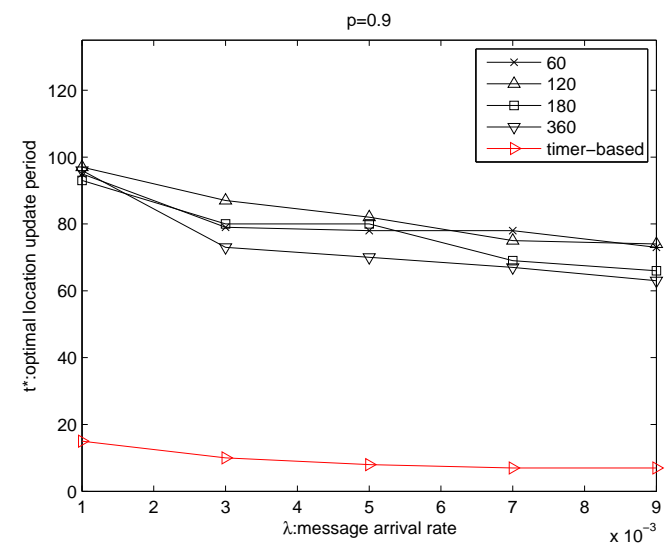

Figure 15: The optimal update period with different low message arrival rates

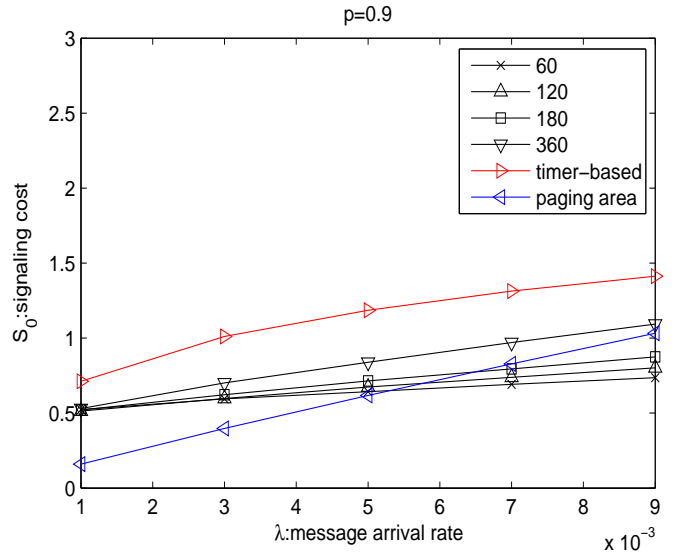

Figure 16: The signaling cost with different low message arrival rates

ically. As a result, the timer based scheme performs poorly in low $\lambda$ scenarios.

As shown in Fig. 17 and 18, we demonstrate the performance in two-tier paging area configurations. With low message arrival rate rate, the signaling cost in two-tier configuration is lower than the performance in one-tier configurations. As discussed previously, the main contributor of the signaing cost is the update cost rather than the broadcast paging cost. The paging area notification signaling cost is higher in the one-tier configuration. Moreover, the timer based scheme must update periodically, so the overall signaling cost is high.

\section{DISCUSSION}

As observed from the simulations, we find that the most suitable location update scheme in different scenarios are different. Depending on the operational parameters, the signaling cost might be dominated by one or more of the cost components: timer based update signaing cost, broadcast paging signaling cost, and paging area notification signaling

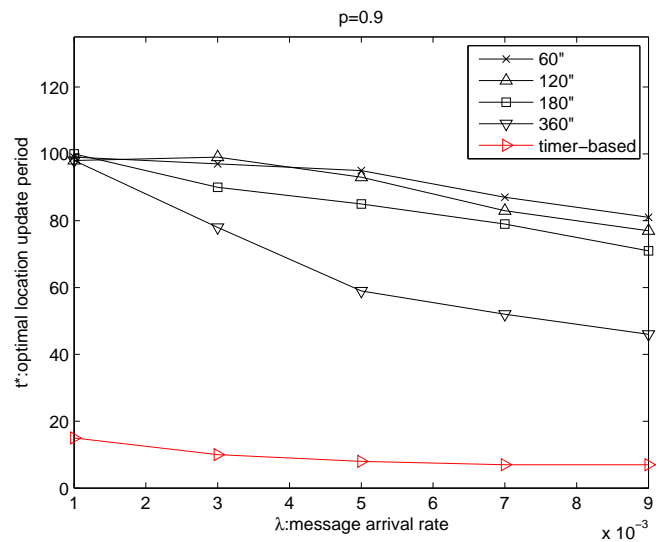

Figure 17: The optimal update period with different low message arrival rates in two-tier paging area configuration

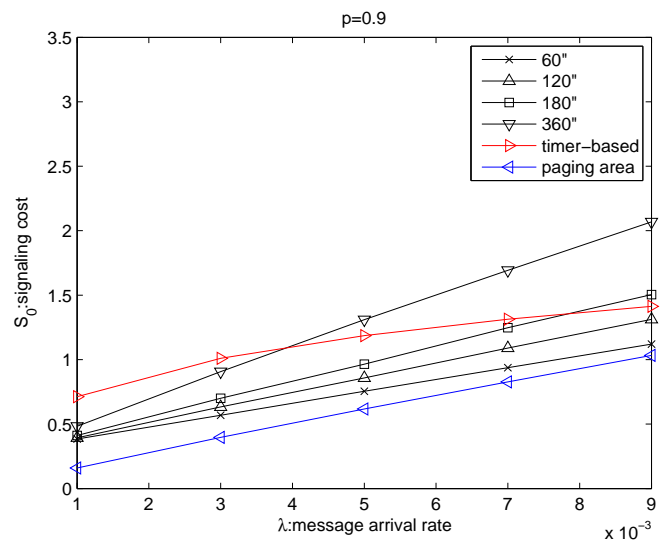

Figure 18: The signaling cost with different low message arrival rate in two-tier paging area configuration 
cost. Some observation on the desireable locaiton update scheme in various sceanarios are described below.

First, the timer-based scheme is suitable when the mobility probability $p$ is small and the message arrival rate $\lambda$ is large. While the mobility probability $p$ is large, which indicates an idle mode MS is more likely to stay in the same paging area, the signaling cost in the timer based scheme is still the same, as the signaling update frequency is based on the timer rather than moving across paging area. The timer based scheme performs well when idle mode MS moves fast and enters active mode frequently. Second, the paging area based update scheme is more suitable when the message arrival rate $\lambda$ is small. In the paging area based update schemes, the paging area configuration applies the same paging area size and pattern. Hence, the signaling cost reduces when message arrives infrequently, which leads to less broadcast paging events. In the paging area based scheme, we can choose use paging area configuration with larger paging area when idle mode MSs move faster. Third, the hybrid schemes usually have better performance in most cases. The optimization of location update timer also effectively reduce overall signaling cost. Among the hybrid location update scheme, the 60-degree one-tier paging area configurations is usually the best. In realistic operational environments, the data message arrival rate often depends on a specific user and its application. The hybrid locaiton update scheme leverages the advantages from the paging area based scheme and timer based scheme. It can apply to different mobility and message arrival rate scenarios.

\section{CONCLUSION}

In this paper, we investigate the location update and paging signaling cost in IEEE 802.16j multihop relay networks. Three types of location update schemes are compared. The timer based location update scheme performs well in scenarios where users move fast and receives message frequently. The paging area based location update scheme performs well in scenarios where users move slow and receives message infrequently. The hybrid update schemes apply both the paging area update concept and the timer update concept. Usually, the hybrid update scheme perform well in all scenarios. In addition, the update timer optimization could effectively enhance the performance of the hybrid scheme.

\section{REFERENCES}

[1] IEEE Standard for Local and Metropolitan Area Networks Part 16: Air Interface for Fixed Broadband Wireless Access Systems. IEEE Std 802.16-2004 (Revision of IEEE Std 802.16-2001), pages 1-857, 2004.

[2] IEEE Standard for Local and metropolitan area networks Part 16: Air Interface for Fixed and Mobile Broadband Wireless Access Systems Amendment 2: Physical and Medium Access Control Layers for Combined Fixed and Mobile Operation in Licensed Bands and Corrigendum 1. IEEE Std 802.16e-2005 and IEEE Std 802.16-2004/Cor 1-2005 (Amendment and Corrigendum to IEEE Std 802.16-2004), pages 1-822, 2006.

[3] IEEE Draft Amendment to IEEE Standard for Local and Metropolitan Area Networks Part 16: Air Interface for Fixed and Mobile Broadband Wireless Access
Systems Multihop Relay Specification. IEEE

Unapproved Draft Std P802.16j/D3, Feb 2008, 2008.

[4] A. Bar-Noy, I. Kessler, and M. Sidi. "Mobile users: To update or not to update?". Wireless Networks, 1(2):175-185, 1995.

[5] H. Wei, S. Ganguly, R. Izmailov, and Z. Haas. Interference-aware IEEE 802.16 WiMax mesh networks. In IEEE 61st Vehicular Technology Conference (VTC 2005-Spring), 2005.

[6] S. Yang, Y. Lin, and Y. Lin. "Performance of Mobile Telecommunications Network With Overlapping Location Area Configuration". IEEE Transactions on Vehicular Technology, 57(2):1285-1292, 2008. 